\title{
ON THE IMPLEMENTATION OF REDUCED GRADIENT METHODS
}

\author{
H. Mukai and E. Polak \\ Department of Electrical Engineering and Computer Sciences \\ and the Electronics Research Laboratory \\ University of California, Berkeley, California 94720
}

\section{ABSTRACT}

Until now, the implementation of reduced gradient methods had to be improvised empirically, since procedures for the truncation of the inner iterations, in the feasibility restoration stage, have not been analyzed with respect to convergence of the overall algorithm. This paper presents an implementation of one reduced gradient method. While retaining all the attractive features of the classical reduced gradient methods, this implementation incorporates, explicitiy, efficient procedures for truncating the inner iterations to a finite number. In the paper, we present the properties of the restoration subalgorithm and we prove the convergence of the new algorithm under fairly general assumptions. 


\section{Introduction}

There are both practical and theoretical difficulties associated with reduced gradient methods as stated in [1], [2], [5], [10]. Both of these difficulties stem from the fact that implementation of these methods has to be improvised empirically, since procedures for the truncation of the infinite inner iteration, in the feasibility restoration stage, have not been analzed with respect to convergence of the overall algorithm. This paper presents an extension of the reduced gradient method in $[6,11]$ : it takes a gradient projection step whener it is not possible to take a reduced gradient step, and it incorporates explicitly the required procedures for truncating the inner iterations to a finite number. We state the properties of the restoration subalgorithms and we prove the convergence of the implementable algorithm under fairly general assumptions.

\section{Algorithm Mode1}

In this section we present a slightly generalized version of the algorithm model for the implementation of approximations presented in [7]. For this purpose, we consider the following abstract

Problem 1: Let $T$ be a closed subset of $\mathbb{R}^{\mathrm{n}}$. Given a nonempty subset $\triangle \subset T$ of desirable points, find a point in $\Delta$. $\square$

We shall denote the Euclidean norm on $\mathbb{R}^{\mathrm{n}}$ by $\|\cdot\|$; and we shall use the notation $B(z, \rho)=\left\{x \in \mathbb{R}^{\mathfrak{n}} \mid\|x-z\| \leq \rho\right\} ; \mathbb{R}_{+}=[0, \infty), \mathbb{R}_{++}=(0, \infty)$. We shall say that a sequence $\left\{z_{i}\right\}_{i=0}^{\infty}$ is compact if it is contained in a compact set.

Our algorithm model makes use of a cost function $f: \mathbb{R}^{n} \rightarrow \mathbb{R}$, an approximating iteration map $A: \mathbb{R}^{\mathrm{n}} \times \mathbb{R}_{+} \rightarrow 2^{\mathbb{R}^{\mathrm{n}}}$, a proximity function $\mathrm{P}: \mathbb{R}^{\mathrm{n}} \rightarrow \mathbb{R}_{+}$which is used to provide a measure of closeness of a point from the set $\mathrm{T}$, and a restoration map $Q: \mathbb{R}^{\mathrm{n}} \times \mathbb{R}_{++} \rightarrow 2^{\mathbb{R}^{\mathfrak{n}}}$, which is used to "drive" points into T. Finally, let $\mathrm{P}: \mathbb{R}_{+} \rightarrow$ ${ }_{2} \mathbb{R}^{\mathfrak{n}}$ be defined by

$$
P(\varepsilon)=\left\{x \in \mathbb{R}^{n} \mid p(x) \leq \varepsilon\right\}
$$

Assumption 1

(i) $\quad f: \mathbb{R}^{\mathrm{n}} \rightarrow \mathbb{R}$ is continuously differentiable.

(ii) $p: \mathbb{R}^{\mathrm{n}} \rightarrow \mathbb{R}_{+}$is continuous, and $P(0)=T$.

(iii) For any $x \in \mathbb{R}^{\mathbb{n}}, \varepsilon>0, Q(x, \varepsilon) \subset P(\varepsilon)$.

(iv) For any $\varepsilon>0$ and $x \in P(\varepsilon), Q(x, \varepsilon)=\{x\}$.

(v) For any $z \in \mathbb{R}^{\mathfrak{n}}$, if the infinite sequences $\left\{x_{i}\right\}$ and $\left\{\varepsilon_{i}\right\}$ satisfy $x_{i} \in Q\left(z, \varepsilon_{i}\right)$, for $i=0,1,2, \ldots$, and $\varepsilon_{i} \rightarrow 0$ as $i \rightarrow \infty$, then $\left\{x_{i}\right\}$ has a finite number of accumulation points, all of which are in $T$.

(vi) For any compact subset $C$ of $\mathbb{R}^{n}$, there exist constants $M, e \in \mathbb{R}_{++}$, such that

$\forall E \in(0, e], \forall x \in C \cap P(\varepsilon), \forall E^{\prime} \in(0, \varepsilon], Q\left(x, \varepsilon^{\prime}\right) \subset B(x, M \varepsilon)$.

(vii) For any $\varepsilon>0$ and $x \in P(\varepsilon), A(x, \varepsilon) \subset P(\varepsilon)$. 
(viii) For any $z \in T \sim \Delta$, there exist $\rho>0, \delta<0$, and $e>0$ such that $\forall E \in(0, e]$, $\forall x \in B(z, \varepsilon)$, $\forall y \in A(x, \varepsilon)$,

$$
f(y)-f(x) \leq \delta<0
$$

\section{Algorithm Mode1}

Parameters: $\gamma>0, \beta \in(0,1)$.

Data: $z_{0} \in \mathbb{R}^{\mathrm{n}}, \varepsilon_{0}>0$.

Step 0: Set $i=0, j=0, \varepsilon=\varepsilon_{0}$.

Step 1: Compute an $\mathrm{x} \in \mathrm{Q}\left(z_{i}, E\right)$.

Step 2: Compute a $y \in A(x, \varepsilon)$.

Step 3: If $f(y)-f(x) \leq-\gamma \varepsilon$, go to step 4; else set $x_{j}=x, \varepsilon=\varepsilon \beta, j=j+1$ and go to step 1 .

Step 4: Set $z_{i+1}=y, i=i+1$ and go to step 1 .

The above Model is an implementation of the limiting case obtained by setting $\varepsilon_{0}=0$ in the data, above. The following result can be deduced from Theorem 1 in [7]. Theorem 1: (i) If the Algorithm Model stops at a particular $z_{i}$ and constructs an infinite sequence $\left\{x_{j}\right\}$, then $\left\{x_{j}\right\}$ has a finite number of accumulation points, all of which are in $\Delta$. (ii) If the Algorithm Model constructs a compact infinite sequence $\left\{z_{i}\right\}$, then any accumulation point of $\left\{z_{i}\right\}$ is in $\Delta$.

\section{A Structure for the Iteration Map A}

In the application we shall discuss in this paper, as well as in some others, the map $A$ introduced in the preceding section has a very complex description and it is quite cumbersome to show directly that it satisfies parts (vii) and (viii) of Assumption 1. Because of this, we introduce an intermediate stage, viz., a structure for A, which guarantees that parts ( $v i i$ ) and (viii) of Assumption 1 are satisfied.

Thus, let $Y$ be an open subset of $\mathbb{R}^{n}$, and let $9,9_{\tilde{N}}, g_{2}$ be two finite sets of indexing parameters, let $\tilde{g}_{1}: x \rightarrow 2^{1}$ and $\tilde{g}_{2}: x \rightarrow 2^{2}$ be two direction selection functions. Finally, let $D_{1}: Y \rightarrow 2^{\mathbb{R}}, D_{2}: \mathbb{R}^{\mathbb{n}} \rightarrow 2^{\mathbb{R}^{\mathrm{n}}}$ be two direction sets defined by

$$
\mathrm{D}_{\mathrm{k}}(\mathrm{x})=\left\{\mathrm{d}_{\mathrm{k}}(\mathrm{x}, \mathrm{I}) \mid \mathrm{I} \in \tilde{\theta}_{\mathrm{k}}(\mathrm{x})\right\}
$$

where for each $x \in \mathbb{R}^{\mathrm{n}}$ the functions $d_{k}(\cdot, I)$ are well defined for all $I \in \tilde{\varphi}_{k}(x)$ on some neighborhood $N_{x}^{k}$ of $x$.

In terms of these, we now define $\phi_{1}: Y \rightarrow \mathbb{R}, \phi_{2}: \mathbb{R}^{\mathrm{n}} \rightarrow \mathbb{R}$ by

$$
\phi_{k}(x)=\max _{I \in \tilde{\varphi}_{k}(x)}\left\langle\nabla f(x), d_{k}(x, I)\right\rangle \quad, k=1,2
$$

and $9_{1}^{*}: \mathrm{Y} \rightarrow 2^{9_{1}}, 9_{2}^{*}: \mathbb{R}^{\mathrm{n}} \rightarrow 2^{9_{2}}$ by 


$$
g_{k}^{*}(x)=\left\{I \in \tilde{Q}_{k}(x) \mid\left\langle\nabla f(x), d_{k}(x, I)\right\rangle=\min _{J \in \tilde{Q}_{k}(x)}\left\langle\nabla f(x), d_{k}(x, J)\right\rangle\right\}, k=1,2
$$

Assumption 2: For $\mathrm{k}=1,2$,

(i) For any $z \in T \cap Y(z \in T$ for $k=2)$ and any $\left.I \in \tilde{9}_{k}(z), p\left(z+\lambda_{k}(z, I)\right) / \lambda\right] \rightarrow 0$ as $\lambda \rightarrow 0$.

(i1) For any $\mathrm{x} \in \mathrm{Y}\left(\mathrm{x} \in \mathbb{R}^{\mathrm{n}}\right.$ for $\left.\mathrm{k}=2\right), \phi_{k}(\mathrm{x}) \leq 0$.

(iii) $\phi_{k}^{-1}(0) \cap T \subset \Delta$.

(iv) For any $\mathrm{x} \in \mathrm{Y}\left(\mathrm{x} \in \mathbb{R}^{\mathrm{n}}\right.$ for $\left.\mathrm{k}=2\right)$ and $I \in \hat{\varphi}_{\mathrm{k}}(\mathrm{x})$, there exists a neighborhood $N$ of $x$ such that $d_{k}(\cdot, I)$ is continuous on $N$.

(v) For any $\mathrm{x} \in \mathrm{Y}\left(\mathrm{x} \in \mathbb{R}^{\mathrm{n}}\right.$ for $\left.\mathrm{k}=2\right)$ there exists a neighborhood $\mathrm{N}$ of $\mathrm{x}$ such that For a11 y $\in \mathrm{N}, \hat{\theta}_{\mathrm{k}}(\mathrm{y}) \subset \hat{9}_{\mathrm{k}}(\mathrm{x})$.

Finally, let $\check{Y} \subset Y$ be such that the closure of $\tilde{Y}$ is also contained in $Y$. We now define the approximating iteration map A in terms of the functions introduced above, which we state in the form of a subalgorithm, as is the case in practice.

\section{Iteration Map A}

Parameters: $\gamma>0, \lambda_{s}>0, \lambda_{\mathrm{e}} \in\left(0, \lambda_{\mathrm{s}} / \varepsilon_{0}\right),{ }^{\dagger} \alpha \in(0,1), \beta \in(0,1)$.

Data: $\mathrm{x} \in \mathbb{R}^{\mathrm{n}}, \varepsilon>0$.

Step 1: If $x \in \tilde{Y}$, set $k=1$; else set $k=2$.

Step 2: Compute $\phi_{k}(x), \varphi_{k}^{*}(x)$, and select any $I \in g_{k}^{*}(x)$.

Step 3: If $\phi_{k}(x) \leq-\gamma \varepsilon$, go to step 4; else, set $A(x, \varepsilon)=\{x\}$, and stop.

Step 4: Set $\lambda=\lambda_{\mathrm{s}}$.

Step 5: If $\lambda \geq \lambda e$, go to step 6; else, set $A(x, \varepsilon)=\{x\}$ and stop.

Step 6: If $f\left(x+\lambda d_{k}(x, I)\right)-f(x) \leq \alpha \lambda \phi_{k}(x)$, go to step 7; else, set $\lambda=\lambda \beta$ and go to step 5 .

Step 7: Compute a $\mathrm{y} \in \mathrm{Q}\left(\mathrm{x}+\lambda \mathrm{d}_{\mathrm{k}}(\mathrm{x}, \mathrm{I}), \varepsilon\right)$.

Step 8: If $f(y)-f(x) \leq \alpha \lambda \phi_{k}(x)$, set $A(x, \varepsilon)=\{y\}$, and stop; else set $\lambda=\lambda \beta$ and go to step 5 .

We now proceed to show that, defined as above, under Assumption 2, A satisfies parts (vii) and (viii) of Assumption 1.

Proposition 1: The functions $\phi_{k}, k=1,2$ are upper semicontinuous.

This result follows directly from Assumptions $1(i)$ and $2(i v), 2(v)$. Next, from the structure of A and Assumption 1(iii) we obtain that A satisfies Assumption 1(vii). Proposition 2: The map A, defined in this section satisfies part (viii) of Assumption 1 .

Proof: Let $z \in T \cap Y \sim \Delta$, and let $C \subset Y$ be a compact neighborhood of $z$. Then by Assumption 1 (iv) and (vi), there exist $M>0$ and $e>0$ such that

$\forall x \in C \cap P(e), \forall \varepsilon \in(0, e], Q(x, \varepsilon) \in B(x, M p(x))$.

${ }_{E_{0}}$ is as defined in the Algorithm Model. 
Next, because $P(e) \cap C$ is a neighborhood of $z$, it follows from Assumption 2(iv) and (v) that there exist $r_{0}>0, r_{1}>0$ and $\bar{\lambda} \in\left(0, \lambda_{s}\right]$ such that $B\left(z, x_{1}\right) \subset Y, B\left(z, r_{1}\right) \subset$ $\mathrm{P}(\mathrm{e}) \cap \mathrm{C}$, and $\forall \mathrm{x} \in \mathrm{B}\left(\mathrm{z}, \mathrm{r}_{0}\right), \tilde{\varphi}_{1}(\mathrm{x}) \in \tilde{Q}_{1}(\mathrm{z})$. Furthermore, $\forall \mathrm{x} \in \mathrm{B}\left(\mathrm{z}, \mathrm{x}_{0}\right), \forall I \in \mathcal{I}_{1}(\mathrm{x})$

$$
\mathrm{x}+\lambda \mathrm{d}_{1}(\mathrm{x}, \mathrm{I}) \in \mathrm{B}\left(\mathrm{z}, \mathrm{r}_{1}\right) \subset \mathrm{P}(\mathrm{e}) \cap \mathrm{C}
$$

It now follows from (6) and (7) that there exists a compact set $U$ such that

$$
\begin{aligned}
V x & \in B\left(z, r_{0}\right), \forall \lambda \in[0, \bar{\lambda}], \forall I \in \hat{\vartheta}_{1}(x), \forall \varepsilon \in(0, e], Q\left(x+\lambda d_{1}(x, I), \varepsilon\right) \\
& \subset B\left(x+\lambda d_{1}(x, I), M p\left(x+\lambda d_{1}(x, I)\right)\right) \subset v
\end{aligned}
$$

Since $f(\cdot)$ is Lipschitz continuous on $U$ because of Assumption $1(i)$, there exists an $\mathrm{L},>$ such that

$$
\begin{aligned}
& \forall \mathrm{x} \in \mathrm{B}\left(\mathrm{x}, \mathrm{x}_{0}\right), \forall \lambda \in[0, \bar{\lambda}], \forall I \in \tilde{\varphi}_{1}(\mathrm{x}), \forall \varepsilon \in(0, \mathrm{e}], \forall \mathrm{y} \in \mathrm{Q}(\mathrm{x}+\lambda \mathrm{d},(\mathrm{x}, \mathrm{I}), \varepsilon), \\
& f(y)-f\left(x+\lambda d_{1}(x, I)\right) \leq L\left\|y-x-\lambda d_{1}(x, I)\right\| L M P\left(x+\lambda d_{1}(x, I)\right)
\end{aligned}
$$

Next, making use of the first order Taylor expansion, we get, $\forall \in\left[0, \lambda_{\mathrm{s}}\right], \forall I \in \Theta_{1}(x)$, $\forall \mathrm{x} \in \mathrm{B}\left(\mathrm{z}, \mathrm{r}_{0}\right)$,

$$
\begin{aligned}
& \begin{array}{l}
\mathrm{B}\left(z, \mathrm{x}_{0}\right), \\
f\left(x+\lambda \mathrm{d}_{1}(\mathrm{x}, \mathrm{I})\right)-f(\mathrm{x})=\lambda\left\langle\nabla f(\mathrm{x}), \mathrm{d}_{1}(\mathrm{x}, \mathrm{I})\right\rangle+\lambda \int_{0}^{1}\left\langle\nabla f\left(z+\mathrm{t} \lambda \mathrm{d}_{1}(z, I)\right)\right.
\end{array} \\
& \left.-\nabla f(z), d_{1}(z, I)\right) d t \leq \lambda \alpha \phi_{1}(x)+\lambda(1-\alpha) \phi_{1}(x) \\
& -\sup _{t \in[0,1]}\left\|\nabla f\left(x+\lambda d_{1}(x, I)\right)-\nabla f(x)\right\| \cdot\left\|d_{1}(x, I)\right\|
\end{aligned}
$$

Now, since $\phi_{1}(*)$ is upper semi-continuous and $\phi_{1}(z)<0$, there exists a $\rho_{z} \epsilon^{*}\left(0, r_{0}{ }^{1}\right.$ such that $\phi_{1}(\mathrm{x}) \leq \frac{1}{2} \phi(\mathrm{z})$ for al1 $\mathrm{x} \in \mathrm{B}\left(\mathrm{x}, \rho_{\mathrm{z}}^{*}\right)_{l^{\prime}}$ and there exists an integer $l_{1} \geq 0$ such that $\forall x \in B\left(z, \rho_{z}^{*}\right), \forall I \in \hat{9}_{1}(x), \forall \lambda \in\left[0, \lambda_{s}{ }^{k}{ }^{1}\right]$

$$
\frac{1}{2}(1-\alpha) \phi_{1}(z)+\sup _{t \in[0,1]}\left\|\nabla f\left(x+t \lambda d_{1}(x, I)\right)-\nabla f(x)\right\| \leq \frac{1}{4}(1-\alpha) \phi_{1}(z) \leq 0
$$

so that $\forall x \in B\left(z, \rho{ }_{z}^{*}\right), \forall I \in \tilde{\rho}_{1}(x), \forall \lambda \in\left[0, \lambda_{s} \beta^{l}{ }^{1}\right]$

$$
f\left(x+\lambda d_{1}(x, I)\right)-f(x) \leq \lambda a \phi_{1}(x)
$$

Because of Assumption $2(i)$ there exists an integer $l_{1 z} \geq \ell_{1}$ such that $\operatorname{LMp}\left(z+\lambda_{1 z} d(z, I)\right)$ $\leq-\frac{1}{8} \lambda(1-\alpha) \phi_{1}(z)$, where $\lambda_{1 z}=\lambda_{s}{ }^{3} 1 z$. It now follows from Assumptions $2(1 v)$, (v) that there exists $\rho_{z} \in\left(0, \rho_{z}^{*}\right]$ such that

$$
\forall \mathrm{x} \in \mathrm{B}\left(z, \rho_{z}\right), \operatorname{LMp}\left(\mathrm{x}+\lambda_{1 z} \mathrm{~d}_{1}(\mathrm{x}, \mathrm{I})\right) \leq-\frac{1}{4} \lambda_{1 z}(1-\alpha) \phi_{1}(z)
$$

It follows from (9) through (11) and (13) that $\forall \mathrm{x} \in \mathrm{B}\left(\mathrm{z}, \rho_{1 z}\right), \forall I \in \tilde{g}_{1}(\mathrm{x})$, $\forall y \in Q\left(z+\lambda_{1 z} d_{1}(x, I), \varepsilon\right), \forall \in \in(0, e)$ 


$$
\begin{aligned}
& f(y)-f(x)=f(y)-f\left(x+\lambda{ }_{1 z} d_{1}(x, I)\right)+f\left(x+\lambda{ }_{1 z} d_{1}(x, I)\right)-f(x) \\
& \leqq \lambda_{1 z}{ }^{\alpha \phi}{ }_{1}(x)+\frac{1}{4} \lambda{ }_{1 z}{ }^{(1-\alpha) \phi}{ }_{1}(z)+\operatorname{LMp}\left(x+\lambda_{1 z}{ }^{d}(x, I)\right) \\
& \leq \lambda_{12}^{\alpha \phi}(x)
\end{aligned}
$$

Now let $e_{1 z} \in(0, e]$ be such that $\lambda_{e_{1 z}} \geq \lambda_{1 z}$, and $\frac{1}{2} \phi_{1}(z) \leq-\gamma e_{1 z}$, let $\varepsilon \in\left(0, e_{1 z}\right]$, and for each $x \in B\left(z, \rho_{1 z}\right)$ and $I \in \tilde{g}_{1}(x)$, let $\tilde{\lambda}_{1}(x, E, I)$ be such that the tests in steps 6 and 8 of the Iteration Map A are satisfied. Then because of (12) and (14), for all $x \in B\left(x, p_{1 z}\right)$, for all $\varepsilon \in\left(0, e_{1 z}\right]$, for all $I \in \tilde{\theta}_{1}(x)$ and for any $y \in$ $\mathrm{Q}\left(\mathrm{x}+\lambda_{1}(\mathrm{x}, \varepsilon, I) \mathrm{d}_{1}(\mathrm{x}, \mathrm{I}), \varepsilon\right)$, we get $\tilde{\lambda}_{1}(\mathrm{x}, \varepsilon, \mathrm{I}) \geqq \lambda_{1 z}$ so that

$$
f(y)-f(x) \leq \alpha \tilde{\lambda}_{1}(x, E, I) \phi_{1}(x) \leq a \lambda_{1 z} \phi_{1}(z) / 2<0
$$

which is of the form of (3).

Now suppose that $z \in T \backsim \Delta$. Then by an identical argument to the one used above, we can show that there exist $\rho_{2 z}>0, e_{2 z}>0$ and a $\lambda_{2 z}>0$ such that for any $x \in$ $B\left(z, \rho_{2 z}\right)$, for any $\varepsilon \in\left(0, e_{2 z}\right]$ for any $I \in \tilde{\rho}_{2}(x)$, and for any $y \in Q\left(x+\tilde{\lambda}_{2}(x, \varepsilon, I) d_{2}(x, I), \varepsilon\right)$, we get

$$
f(y)-f(x) \leq \alpha \lambda_{2 z} \phi_{2}(z) / 2<0
$$

Consequently, since the closure of $\tilde{Y}$ is contained in $Y$, we now get the following conclusion. Suppose $z \in T \sim \Delta$ satisfies $z \notin Y$, then, from the above, there exists a ball $B\left(z, \rho_{2 z}\right)$, a $\lambda_{2 z}>0$ and an $e_{2 z}>0$ such that $B\left(z, \rho_{2 z}\right) \cap \tilde{Y}=\phi$, and $\forall x \in B\left(z, p_{2 z}\right)$, $\forall \varepsilon \in\left(0, e_{2 z}\right]$, the map A sets $y=x+\tilde{\lambda}_{2}(x, \varepsilon, I) d_{2}(x, I)$, with $I \in \tilde{\rho}_{2}^{*}(x)$, and (16) holds for all such $y$. Next, suppose that $z \in T \sim \Delta$ and $z \in Y$. Then, from the above, there exists a $p_{z}=\min \left\{p_{1 z}, \rho_{2 z}\right\}$ and an $e_{z}=\min \left\{e_{1 z}, e_{2 z}\right\}$, such that for all $x \in$ $\mathrm{B}\left(z, \rho_{\mathrm{z}}\right)$, and all $\varepsilon \in\left(0, \mathrm{e}_{\mathrm{z}}\right]$, the map A sets $\mathrm{y}=\mathrm{x}+\tilde{\lambda}_{1}(\mathrm{x}, \varepsilon, I) \mathrm{d}_{1}(\mathrm{x}, \mathrm{I})$, with $\mathrm{I} \in \mathcal{G}_{1}^{*}(\mathrm{x})$, or $y=x+\tilde{\lambda}_{2}(x, \varepsilon, I) d_{2}(x, I)$, with $I \in Q_{2}^{*}(x)$, depending on whether $x \in \tilde{Y}$ or not, but in either event, we get

$$
f(y)-f(x) \leq \frac{1}{2} \alpha \max \left\{\lambda_{1 z^{\phi}}(z), \lambda_{2 z^{\phi}}{ }_{2}(z)\right\}<0
$$

$\forall x \in B\left(z, \rho_{z}\right), \forall \varepsilon \in\left(0, e_{z}\right], \forall y \in A(x, \varepsilon)$. Thus, the iteration map $A$, defined in this section satisfies Assumption 1(viii), which completes our proof. I

\section{An Implementable Reduced Gradient Method}

We shall now present an implementable generalization of the reduced gradient method analyzed by Luenberger [6] (Ex. 18, p. 275) for the case of linear equality constraints only. Thus, we sha1l consider the problem

$$
\min \{f(x) \mid g(x)=0, x \geq 0\}
$$

where $\mathrm{f}: \mathbb{R}^{\mathfrak{n}} \rightarrow \mathbb{R}^{1}, g: \mathbb{R}^{\mathrm{n}} \rightarrow \mathbb{R}^{\mathbb{m}}(\underline{m} \leq \mathrm{n})$. 
We sha1l denote the components of a vector by superscripts; given $x \in \mathbb{R}^{\mathrm{n}}$, $x^{+} \in \mathbb{R}_{+}^{n}, x^{-} \in \mathbb{R}_{-}^{n}$ are defined componentwise by $x^{+i}=\max \left\{0, x^{i}\right\}, x^{-i}=\min \left\{0, x^{i}\right\}$, $i=1,2, \ldots, n$, respectively; $T=\left\{x \in \mathbb{R}^{\mathrm{n}} \lg (\mathrm{x})=0, \mathrm{x} \geq 0\right\}$.

\section{Assumption 3:}

(i) The function $f$ is continuously differentiable.

(ii) The function $g$ is twice continuously differentiable.

(iii) For all $x \in \mathbb{R}^{n}$, the pair of matrices $\left(\frac{\partial g(x)}{\partial x}, I_{n}\right)$, where $I_{n}$ is the $n \times n$ identity matrix, satisfies the LI condition [9], viz., $\frac{\partial g(x)^{T}}{\partial x} \psi+I_{n} \nu=0$ and $\nu \geqq 0$, implies that $\psi=0$ and $\nu=0$. $\quad \square$

Proposition 3: The set $\mathrm{T}$ satisfies the Kuhn-Tucker constraint qualification (see Theorem 3.3.17 in [4]). 口

Proposition 4: The system $\frac{\partial g(x)}{\partial x} v=a, v \geq b$, has a solution for any $x \in \mathbb{R}^{n}, a \in \mathbb{R}^{\mathrm{m}}$, $b \in \mathbb{R}^{\mathfrak{n}}$ if and only if Assumption 3 (iii) is satisfied (see [9]).

The algorithm in this section seeks Kuhn-Tucker points, which we define as being points $\mathrm{x} \in T$ such that there exist multipliers $v \geq 0$ and $\psi$ satisfying $-\nabla f(x)+$ $\frac{\partial g(x)^{T}}{\partial x} \psi+\nu=0$, and $\langle\nu, x\rangle=0$. We identify $\Delta$ with the set of Kuhn-Tucker points.

Now, given $I=\left\{\hat{i}_{1}, i_{2}, \ldots, i_{\ell}\right\} \subset\{1,2, \ldots, n\}\left(i_{1}<i_{2}<\ldots<i_{\ell}\right)$ and $x \in \mathbb{R}^{n}$, we define $x_{I} \in \mathbb{R}^{\ell}$, the $m \times \ell \operatorname{matx} i x \frac{\partial g(x)}{\partial x_{1}}$ and $\nabla_{I} f(x) \in \mathbb{R}^{l}$ by

$$
\begin{aligned}
& x_{I}=\left(x^{i_{1}}, x^{i_{2}}, \ldots, x^{i} b^{T}\right. \\
& \frac{\partial g(x)}{\partial x_{1}}=\left[\begin{array}{ccc}
\frac{\partial g^{1}(x)}{i_{1}} & \cdots & \frac{\partial g^{1}(x)}{i_{l}} \\
\vdots & & \vdots \\
\frac{\partial \mathrm{g}^{1}(x)}{i_{1}} & \cdots & \frac{\partial g^{m}(x)}{\partial x_{l}}
\end{array}\right] \\
& \nabla_{I} f(x)=\left(\frac{\partial f(x)}{\partial x_{1}}, \frac{\partial f(x)}{\partial x^{2}}, \ldots, \frac{\partial f(x)}{\partial x_{\ell}}\right)^{T}
\end{aligned}
$$

Next, we define $\theta_{2}$ to be a singleton and $\theta_{1}$ to be the set

$$
y_{1}=\{\mathrm{I} \subset\{1,2, \ldots, \mathrm{n}\}|| I \mid=\mathrm{n}-\mathrm{m}\}
$$

where $|I|$ denotes the cardinaIity of $I$. For any $Q_{1}^{I} \in Q_{1}$ we shall $0_{2}^{\text {denote }}$ its complement in $\{1,2, \ldots, n\}$ by $\bar{I}$. And we define $\tilde{\varphi}_{1}: \mathbb{R}^{n} \rightarrow 2, \tilde{\varphi}_{2}: \mathbb{R}^{n} \rightarrow 2$ and $p: \mathbb{R}^{n} \rightarrow \mathbb{R}_{+}$ by

$$
\begin{aligned}
& \tilde{\rho}_{1}(x)=\left\{I \in \varphi_{1} \mid x^{i} \leq x^{j} \forall i \in I, \forall j \in \vec{I}\right\}^{\dagger} \\
& \tilde{g}_{2}(x) \equiv 9_{2}
\end{aligned}
$$

\footnotetext{
${ }^{T}$ This is the variable selection scheme used by Zangwill [11] and Luenberger [6].
} 


$$
p(x)=\left(\|g(x)\|^{2}+\left\|x^{-}\right\|^{2}\right)^{1 / 2}
$$

Note that $p$, as defined above, satisfies Assumption 1(ii).

We shall also need the following quantities:

$$
\begin{aligned}
& p_{1}(x) \triangleq \frac{1}{2}\|g(x)\|^{2} \\
& p_{2}(x) \triangleq \frac{1}{2} p(x)^{2}
\end{aligned}
$$

and for any $I \in g_{1}, E(I)$ will denote the $n \times n$ permutation matrix satisfying

$$
x=E(I)\left(\begin{array}{c}
x_{\bar{I}} \\
x_{I}
\end{array}\right)
$$

and $t: \mathbb{R}^{\mathrm{n}} \times 9_{1} \rightarrow \mathbb{R}_{+}$is defined by

$$
t(x, I)=\left|\operatorname{det} \frac{\partial g(x)}{\partial x_{\bar{I}}}\right|
$$

Referring to Sec. 3, we see that we must define the restoration map $Q$ before we can define the iteration on map A. Let $v_{1}: \mathbb{R}^{\mathrm{n}} \times \theta_{1} \rightarrow \mathbb{R}^{\mathrm{n}}$ and $\mathrm{v}_{2}: \mathbb{R}^{\mathrm{n}} \rightarrow \mathbb{R}^{\mathrm{n}}$ be defined by

$$
\begin{aligned}
& v_{1}(x, I)=-E(I)\left[\begin{array}{l}
\frac{\partial g(x)^{-1}}{\partial x \bar{I}} \\
-\frac{g}{0}
\end{array}\right] \quad \text { whenever } t(x, I) \neq 0 \\
& v_{2}(x, I)=\arg \min _{v \in \mathbb{R}^{n}}\left\{\|v\|^{2} \mid g(x)+\frac{\partial g(x)}{\partial x} v=0, x+v \geqq 0\right\}
\end{aligned}
$$

We need not define $v_{1}$ at $(x, I)$ such that $t(x, I)=0$.

Restoration Subalgorithm $\mathrm{g}_{j}: \mathbb{R}^{\mathrm{n}} \times \mathbb{R}_{++} \times \varphi_{1} \rightarrow \mathbb{R}^{\mathrm{n}}, j=1,2$

Parameters: $\quad a>0, \alpha \in(0,1), \beta \in(0,1)$.

Data: $\quad z \in \mathbb{R}^{\mathrm{n}}, \quad \varepsilon>0, \quad I \in \Theta_{1}$ (when $j=1$ ), $j$.

Step 0: If $j=2$, go to step 6 ; else go to step 1 .

Step 1: If $p(z) \leq \varepsilon$, set $q_{1}(z, \varepsilon, I)=z$ and stop; else, go to step 2 .

Step 2: Set $x_{0}=z$ and $k=0$.

Step 3: If $t\left(x_{k}, I\right)>a$ and $x_{k} \geqq 0$, go to step 4; else, go to step 6 .

Step 4: If $p\left(x_{k}\right) \leq \varepsilon$, set $q_{I}(z, \varepsilon, I)=x_{k}$ and stop; else go to step 5 .

Step 5: Set

$$
x_{k+1}=x_{k}+\beta^{l} k_{v_{1}}\left(x_{k}, I\right)
$$

where $l_{k} \geq 0$ is the smallest integer satisfying

$$
p_{1}\left(x_{k}+\beta^{\ell_{k}} v_{1}\left(x_{\ell}, I\right)\right) \leq\left(1-\alpha \beta^{l_{k}}\right) p_{1}\left(x_{k}\right),
$$

set $k=k+1$ and go to step 3 .

Step 6: Set $x_{0}=z$ and $k=0$.

Step 7: If $p\left(x_{k}\right) \leq \varepsilon$, set $q_{j}(z, \varepsilon, I)=x_{k}$ and stop; else, go to step 8 . 
Step 8: Set

$$
x_{k+1}=x_{k}+\beta v_{2}^{k}\left(x_{k}\right)
$$

where $l_{k} \geq 0$ is the smallest integer satisfying

$$
\mathrm{p}_{2}\left(\mathrm{x}_{\mathrm{k}}+\beta^{l} \mathrm{k}_{2}\right) \leq\left(1-\alpha \beta^{l} \mathrm{k}^{\mathrm{k}}\right) \mathrm{p}_{2}\left(\mathrm{x}_{\mathrm{k}}\right)
$$

set $k=k+1$ and go to step 7. I

We now define $Q: \mathbb{R} \times \mathbb{R}_{++} \rightarrow \mathbb{R}^{\mathrm{n}}$ by

$$
Q(x, \varepsilon)=\left\{q_{j}(x, \varepsilon, I) \mid I \in \tilde{Q}_{1}(x), j=1,2\right\}
$$

Since $q$ is eicher a globalized Newton's method in $\mathbb{R}^{\mathbb{T}}$ or a globalized Newton's method in $\mathbb{R}^{\mathrm{n}}$, we see that Assumption $3(i i),(i i i)$ and the following Assumption 4 ensures that $Q$, as defined in (33), satisfies Assumption 1(iii), (iv), (v) and (vi) (for a proof, see [7]).

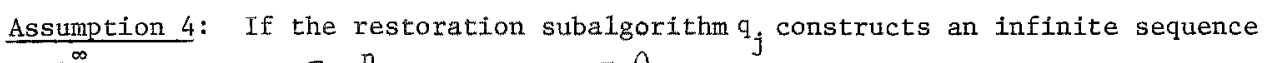
$\left\{x_{k}\right\}_{k=0}^{\infty}$ for some $z \in \mathbb{R}^{\mathbb{n}}, \varepsilon>0$ and $I \in \Theta_{1}$, then the constructed sequence $\left\{x_{k}\right\}$ has an accumulation point. $\square$

Next, we define the sets $Y$ and $\tilde{Y}$, as follows. Let $\omega_{1}>0, \omega_{2}>0$ be two parameters, then

$$
\begin{aligned}
& Y=\left\{x \in \mathbb{R}^{\mathrm{n}} \mid t(x, I)>0, \text { wI } \in \tilde{Q}_{1}(x)\right\} \\
& \tilde{Y}=\left\{x \in \mathbb{R}^{\mathrm{n}} \mid t(x, I)>\omega_{1}, \forall I \in \mathscr{I}(\mathrm{K})\right\}
\end{aligned}
$$

where $\mathcal{T}: \mathbb{R}^{\mathrm{n}}+2^{9}$ is defined by

$$
\mathcal{K}(x)=\left\{x \in g_{1} \mid x^{i} \leq x^{j}+\omega_{2}, \forall i \in I, \forall j \in \bar{I}\right\}
$$

Clearly, as defined in (34), $Y$ is an open set and since $\omega_{2}>0, \mathcal{G}_{(x)} \supset \tilde{\varphi}_{1}(\mathrm{x})$, so that $\tilde{\mathrm{Y}} \subset \mathrm{Y}$.

Proposition 5: The closure of the set $\tilde{Y}$ (as defined in (35)) is contained in $Y$ (as defined in (34)).

Proof: Let $\left\{x_{i}\right\}_{i=0}^{\infty}$ be a sequence in $\tilde{Y}$ converging to a point $x^{*}$. Then, for some $i_{0}$, $\widetilde{K}\left(x_{i}\right) \supset \tilde{g}_{1}\left(x^{*}\right)$ for all $i \geq i_{0}$, and hence we must have $t\left(x^{*}, I\right) \geq w_{1}>0$ for all
$I \in g_{1}\left(x^{*}\right)$. Hence $x^{*} \in Y$.

We now proceed to define the function $d_{1}(\cdot, \cdot)$. For every $x \in Y$ and $I \in \oint_{1}(x)$, we define the reduced gradient by

$$
r(x, I)=\nabla_{I} f(x)-\frac{\partial g(x)}{\partial x_{I}} \frac{\partial g(x)^{-1}}{\partial x_{\bar{I}}} \nabla_{\bar{I}} f(x)
$$

Next, we define the vector $d_{1}(x, I)_{I}$ componentwise by

$$
d_{1}(x, I) \frac{i}{I}=\left\{\begin{array}{l}
-r(x, I) \text { if }-r^{i}(x, I)>0 \\
-\max \left\{x_{I}^{i}, 0\right\} r^{i}(x, I) \text { otherwise }
\end{array}\right.
$$


and the vector $d_{1}(x, I) \bar{I}$ by

$$
d_{1}(x, I)_{\bar{I}}=-\frac{\partial g(x)^{-1}}{\partial x_{\bar{I}}} \frac{\partial g(x)}{\partial x_{1}} d_{1}(x, I) I
$$

Finally, we define $a_{1}(x, I)$ by

$$
d_{1}(x, I)=E(I)\left(\begin{array}{l}
d_{1}(x, I)-\bar{I} \\
d_{1}(x, I)_{I}
\end{array}\right)
$$

The following hypothesis corresponds to the nondegeneracy assumption of linear programing.

Assumption 5: For any $\mathrm{x} \in \mathrm{T}$ and $\mathrm{I} \in \tilde{\rho}_{1}(\mathrm{x}), \mathrm{x}_{\overline{\mathrm{I}}}>0$.

Lemma 2: Assumption 2 is satisfied for $k=1$.

Proof: (i) Let $z \in T \cap Y$, and let $I \in \tilde{\theta}_{1}(z)$. Then $d_{1}(z, I)$ is well defined. Next, because of (38b),

$$
\frac{\partial g(z)}{\partial x} d_{1}(z, I)=\frac{\partial g(z)}{\partial x_{I}} d_{1}(z, I)_{I}+\frac{\partial g(z)}{\partial x_{\bar{I}}} d_{1}(x, I) \bar{I}_{\bar{I}}=0
$$

Hence, since $g(z)=0$, we get

$$
g\left(z+\lambda d_{1}(z, I)\right)=\lambda \int_{0}^{1}\left[\frac{\partial g}{\partial x}\left(z+s d_{1}(z, I)\right)-\frac{\partial g(z)}{\partial x}\right] d_{1}(z, I) d s
$$

and hence, since $g$ is twice continuously differentiable, there exists an $L \in \mathbb{R}_{H}$ such that $\forall \lambda \in[0,1]$,

$$
\left\|g\left(z+\lambda d_{1}(z, I)\right)\right\| \leq \lambda^{2} L\left\|d_{1}(z, I)\right\|^{2}
$$

Next, since by Assumption $5, z_{\bar{I}^{\prime}}>0$, there exists a $\lambda_{0}>0$ such that $z_{\bar{I}}+\lambda d_{1}(z, I) \bar{I}$ $\geqq 0$ for all $\lambda \in\left[0, \lambda_{0}\right]$. Next since $d_{1}(z, I)_{I}^{i} \geqq 0$ for all $i$ such that $z_{I}^{\frac{1}{1}}=0$, it follows that there exists a $\bar{\lambda}_{0} \in\left(0, \lambda_{0}\right]$ such that $z+\lambda d_{1}(z, I) \geq 0$ (i.e., $\left(z+\lambda d_{1}(z, I)\right)^{-}$ $=0$, for all $\left.\lambda \in\left[0, \bar{\lambda}_{0}\right]\right)$. Hence, for all $\lambda \in\left[0, \bar{\lambda}_{0}\right], p\left(z+\lambda d_{1}(z, I)\right)=\| g\left(z+\lambda d_{1}(z, I) \|\right.$, and the desired result now follows from (42).

(ii) By direct calculation, for any $x \in \mathrm{y}$,

$$
\phi_{1}(x)=\max _{I \in \hat{O}_{1}}\left\langle\nabla f(x), d_{1}(x, I)\right\rangle
$$

Now, for any $I \in \hat{\varphi}_{1}(x)$,

$$
\begin{aligned}
\left\langle\nabla f(x), d_{1}(x, I)\right\rangle & =\left\langle\nabla_{I} f(x), d_{1}(x, I)_{I}\right\rangle+\left\langle\nabla_{\bar{I}} f(x), d_{1}(x, I) \bar{I}_{\bar{I}}\right\rangle \\
& =\left\langle r(x, I), d_{1}(x, I)_{I}\right\rangle \leq 0
\end{aligned}
$$

and we are done.

(iii) Suppose $z \in T \cap Y$ is such that $\phi_{1}(z)=0$. Then there exists an $I \in \tilde{\theta}_{1}(z)$ such that $\left\langle\nabla f(z), d_{1}(z, I)\right\rangle=\left\langle r(z, I), d_{1}(z, I) I_{I}\right\rangle=0$. Let $\psi=\frac{\partial g(z)}{\partial x_{\bar{I}}}{ }^{-1 T} \nabla f_{\bar{I}}(z), v_{I}=r(z, I)$ 
and $v_{I}=0$. Then we get $v=E(I)\left(\begin{array}{c}v_{I} \\ v_{I}\end{array}\right) \geq 0$.

$$
\begin{aligned}
& -\nabla_{I} f(z)+\frac{\partial g(z)^{T}}{\partial x_{\tilde{I}}} \psi+\psi_{I}=0 \\
& -\nabla_{I} f(z)+\frac{\partial g(z)^{T}}{\partial x_{I}} \psi+\nu_{I}=0
\end{aligned}
$$

and $\langle\nu, z\rangle=0$, so that $z$ is a Kuhn-Tucker point, i.e., $z \in \Delta$.

(iv) This follows from the continuity of $t$.

(v) Let $x \in Y$ be arbitrary and let $i_{1} \neq i_{2} \neq \ldots \neq i_{n}$ in $\{1,2, \ldots, n\}$ be such that $\mathrm{x}^{i_{1}} \leq \mathrm{x}^{i_{2}} \leq \ldots \leq \mathrm{x}^{i_{n}}$. There must exist two integers $\alpha, \beta, \alpha \leq \mathrm{n}-\mathrm{m} \leq \beta$ such that $x^{i_{1}} \leq x^{i_{2}} \leq x^{i_{3}} \ldots \leq x^{i_{\alpha-1}} x^{i_{\alpha}}=x^{i} \alpha+1=\ldots=x^{i_{\beta}}<x^{i_{\beta+1}} \leq \ldots \leq x^{i^{n}}$, and hence $\tilde{g}_{1}(x)=\left\{I \in\left\{i_{1}, i_{2}, \ldots, i\right\}|| I \mid=n-m\right.$ and $\left.i_{1}, i_{2}, \ldots, i_{\alpha_{-1}} \in I\right\}$. By inspection, there exists a neighborhood $N$ of $x$ such for all $y \in N, y^{i_{1}}, y^{2}, \ldots, y^{i_{\alpha-1}}<y^{i_{\alpha}}, \ldots, y^{i_{\beta}}<$ $\mathrm{y}^{i_{\beta+1}}, \ldots, \mathrm{y}^{i_{n}}$, and hence $\tilde{\varphi}_{1}(\mathrm{y}) \subset \tilde{Q}_{1}(\mathrm{x})$ for all $\mathrm{y} \in \mathrm{N}$. This complete our proof. Next, for any $x \in \mathbb{R}^{\mathrm{n}}$, and any $I$ in $\tilde{\hat{G}}_{2}(x)$ we define $d_{2}(x, I)$ by

$$
d_{2}(x, I)=\arg \min _{d \in \mathbb{R}^{n}} \|\left\{\nabla f(x)+d \|^{2} \mid \frac{\partial g(x)}{\partial x} d=0, x^{+}+d \geq 0\right\}
$$

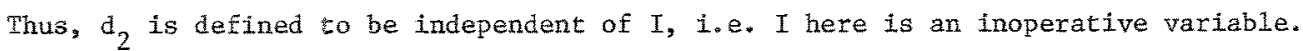
The formula (46) is that of gradient projection, as discussed in [7] and the fact that Assumption 2 is satisfied for $k=2$ follows directly from the results in [7]. Consequently, we can summarize our results as follows.

Theorem 2: The iteration map A defined for problem (18) by (22), (23a, b), (24) (34) and (46) satisfies the conditions (vii) and (viii) of Assumption 1, the function p defined in (24) satisfies the conditions (ii) of Assumption 1 and the map Q, defined by (33) satisfies the conditions (iii) through (vi) of Assumption 1. I Corollary: Consider problem (18) and the implementable reduced gradient algorithm defined by the Algorithm Mode1, the Iteration map A subprocedure of Sec. 3, with all quantities defined as in this section for problem (18) and $Q$ defined as in (33). Then the conclusions of Theorem 1 apply to any sequences $\left\{z_{i}\right\}$ and $\left\{x_{j}\right\}$ that this algorithm has constructed. 


\section{REFERENCES}

[1] J. Abadie and J. Carpentier, Generalization of the Wolfe Reduced Gradient Method for the Case of Nonlinear Constraints," in Optimization, ed. by R. Fletcher, Academic Press, 1969.

[2] J. Abadie and J. Guigou, "Numerical Experiments with the GRG Method," in Integer and Nonlinear Programming, ed. by J. Abadie, North-Holland Pub. Co., Amsterdam, 1970.

[3] L. Armijo, "Minimization of Functions having Continuous Partial Derivatives," Pacific J. Math., vo1. 16, pp. 1-3, 1966.

[4] M. D. Canon, C. D. Cullum, Jr. and E. Polak, Theory of Optimal Control and Mathematical Programming, MeGraw-Hill, 1970.

[5] D. Babay and D. G. Luenberger, "Efficiently Converging Minimization Methods Based on the Reduced Gradient," SIAM Journal on Control, in press.

[6] D. G. Luenberger, Introduction to Linear and Nonlinear Programaing, AddisonWesley Pub. Co., 1973.

[7] H. Mukai and E. Polak, "On the Use of Approximations in Algorithms for Optimization Problems with Equality and Inequality Constraints," Electronics Research Laboratory Memorandum No. 489, University of California, Berkeley, November 1974.

[8] E. Polak, Computational Methods in Optimization, Academic Press, 1971.

[9] S. M. Robinson, "Extension of Newton's Method to Mixed Systems of Nonlinear Equations and Inequalities," Tech. Sum. Rept. no. 1161, Mathematical Research Center, University of Wisconsin, 1.971.

[10] P. Wolfe, "Methods for Nonlinear Constraints," in Nonlinear Programming, ed. by J. Abadie, North-Holland Pub. Co., Amsterdam, 1967.

[11] W. I. Zangwi11, Nonlinear Frogramming: A Unified Approach, Prentice-Ha11, Englewood Cliffs, New Jersey, 1969. 